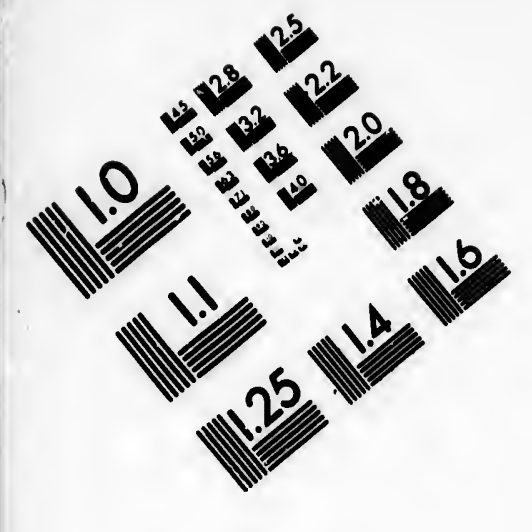

IMAGE EVALUATION

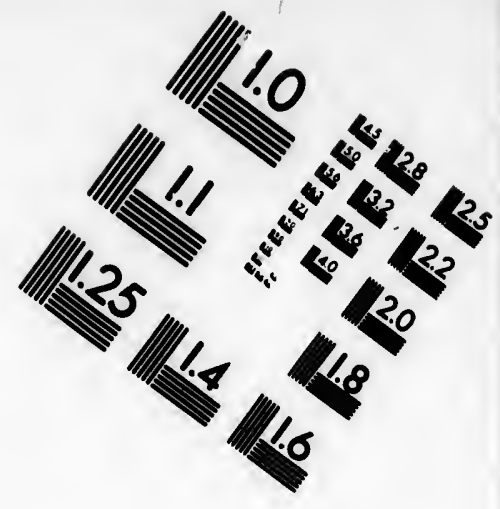

TEST TARGET (MT-3)
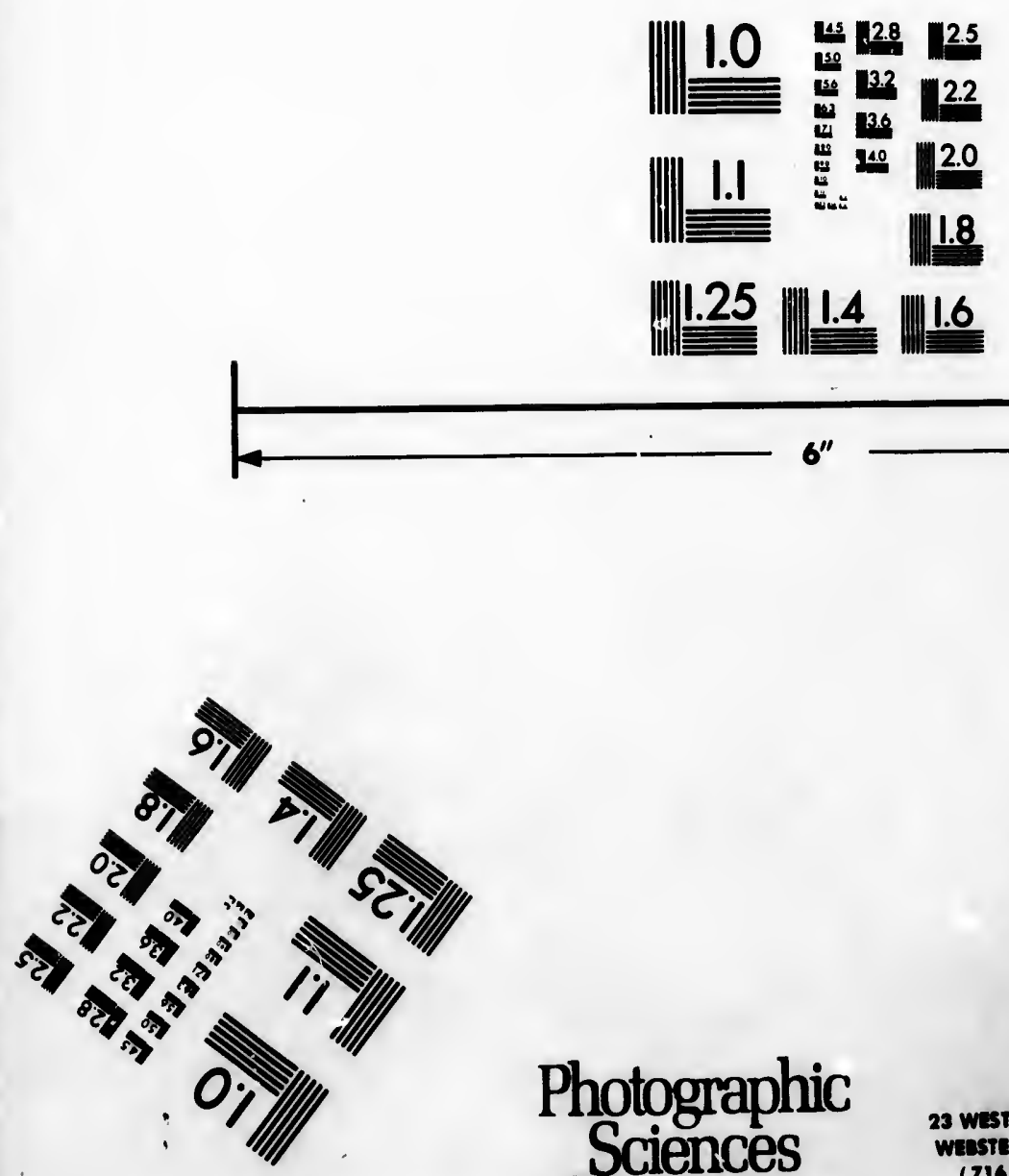

Photographic

Sciences

23 WEST MAN STREST WESSTER, N.Y. 14580

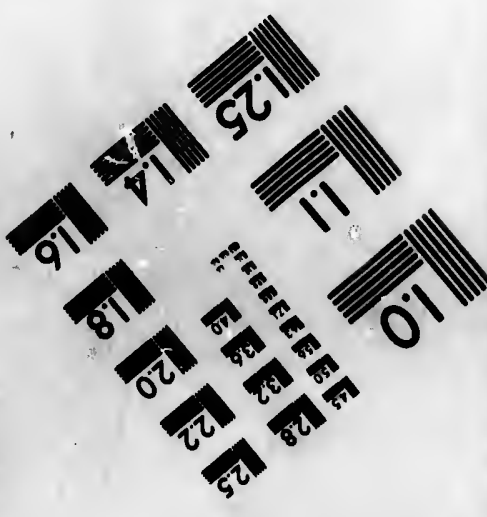
Corporation 


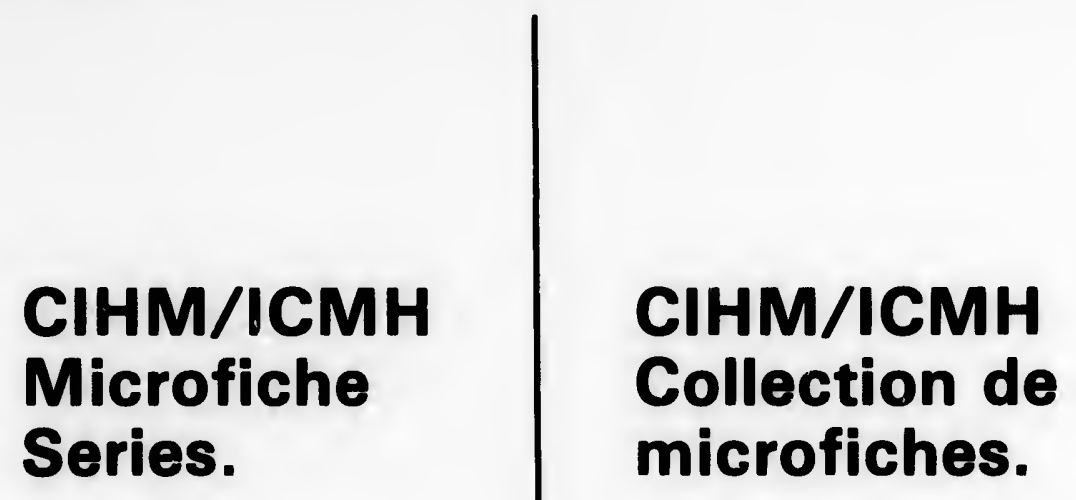

Canadian Institute for Historical Microreproductions / Institut canadien de microreproductions historiques
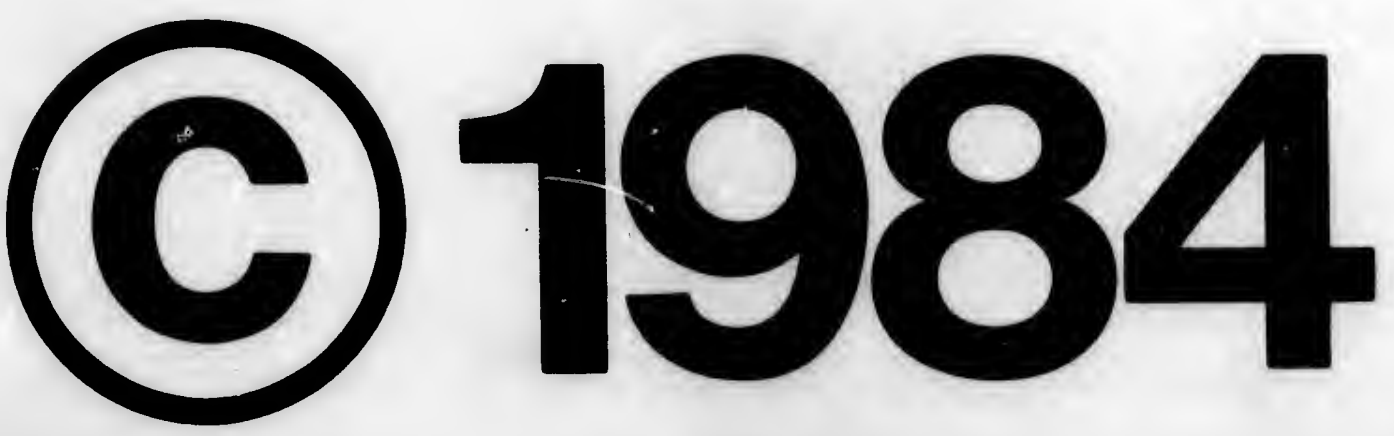
The Institute has attempted to obtain the best original copy available for filming. Features of this copy which may be bibliographically unique. which may alter any of the images in the reproduction, or which may significantly change the usual method of filming, are checked be: ow.

\section{Coloured covera/}

Couverture de couleur

\section{Covers damaged/}

Couverture endommagé

Covers reatored and/or laminated/

Couverture restaurbe ot/ou pellicules

Cover title misaing/

Le titre de couverture manque

Coloured maps/

Cartes géographiques en couleur

Coloured ink (i.e. other than blue or black)/

Encre de couleur (1.e. autre que bleue ou noire)

Coloured plates and/or illuatrationa/

Planches et/ou Illustrations en couleur

Bound with other material/

Relie avec d'autres documents

Tight binding may cause shadows or distortion along interior margin/

Lareliure serrée peut causer de l'ombre ou de la distortion le long de la marge intérieure

Blank leaves added during restoration may appear within the text. Whenever possible, these have been omitted from filming/

II se peut que certaines pages blanches ajout6es lors d'une restauration apparaiasent dans le texte. mais, lorsque cela était possible, ces pages n'ont pas ote filmées.
L'Institut a microfilmb le moilleur exemplaire qu'll lui a ót'́ possible de se procurer. Les dótaila de cet exemplaire qui sont peut-Otre uniques du point de vue bibliographique, qui peuvent modifier une image reproduite, ou qui peuvent exiger une modification dens la móthode normale de filmage sont indiqubs ci-dessous.

\section{Coloured pages/}

Pages de couleur

Pages damaged/

Pages endommegbes

Pages restored and/or laminated/

Pages restaurbes ot/ou pelliculbes

Pages discoloured, stained or foxed/

Pages décolorbes, tachetbes ou piquées

Pages detached/

Pages dotachées

\section{Showthrough/}

Transparence

Quality of print variea/

Qualité insgale de l'impreasion

Includes supplementary materiai/

Comprend du matóriel supplómentaire

\section{Only edition available/}

Seule Edition disponible

Pages wholly or partially obscured by errata slips, tissues, otc., have been refilmed to ensure the best possible image/

Les pages totalement ou partiellement obscurcies par un faulllet d'errata, une pelure. etc., ont btó filmbes nouveau de façon a obtenir la meilloure image possible.

This item is filmed at the reduction ratio chocked below/ Ce document est filmb au taux de róduction indiqub ci-dessous.

$10 x$ $14 \mathrm{X}$ $18 x$ $22 x$ $28 x$ $30 x$

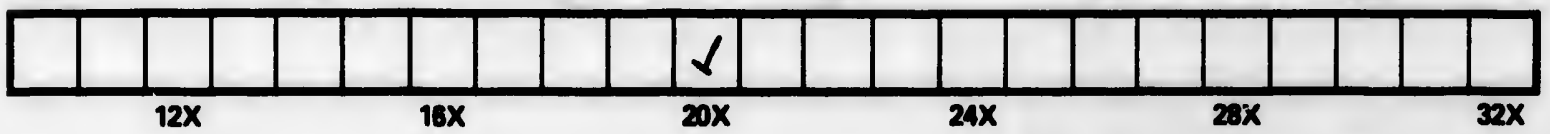


The copy fllmed here has been reproduced thenks to the generosity of:

\section{National Library of Canada}

The imeges appearing here are the best quelity poselble considering the condition and legibility of the original copy and in keeping with the filming contract speciflcetions.

Original copies in printed paper covers are filmed beginning with the front cover and onding on the last page with a printed or lilustreted impression, or the back cover when appropriate. All other original coples are filmed beginning on the first page with a printed or illustrated impresslon, and onding on the last page with a printed or illustrated Impression.

The last recorded frame on each microfiche shall contain the symbol $\rightarrow$ Imeening "CONTINUED"), or the symbol $\nabla$ (meaning "END"). whichover applies.

Meps, plates, cherts, otc., may be filmed at different reduction rotios. Those too large to be entirely included in one exposure are filmed beginning in the upper loft hand corner, loft to right and top to bottom, as many fremes as required. The following diagrams illustrate the mothod:
L'oxempiaire filmo fut reproduit grace o la gónórosité do:

Bibliothdque nationale du Canada

Les images suivantes ont 6́t6 reprodultes avec lo plus grand soin, compte tenu de la condition ot do la nettote de l'exempleire filmé, ot on conformite evec los conditions du contrat de filmago.

Les exemplaires originaux dont la couverture on papier est imprimbe sont filmés en commençant par lo premier plat et en terminant soit par la dernidre page qui comporte une empreinte d'impression ou d'illustration, soit par lo second plat, selon le ces. Tous les autres exemplaires originaux sont filmós on commençant par la premidre page qui comporte une empreinte d'impression ou d'illustration ot en terminant par la dernidre page qui comporte une telle ompreinte.

Un des symboles suivente apparaitra sur la dernibre image de cheque microfiche, selon le cas: le symbole $\rightarrow$ signifie "A SUIVRE", le symbole $\nabla$ signifie "FIN".

Les cartes, planches, tableaux, etc., peuvent otre filmós à des taux de réduction diffórents. Loreque lo document est trop grend pour étre reproduit on un soul clichs, il est filme d partir de l'angle supérieur gauche, de gauche droite. ot de haut on bas, on prenant le nombre d'images nócessaire. Les diagrammes suivants illustrent la methode.
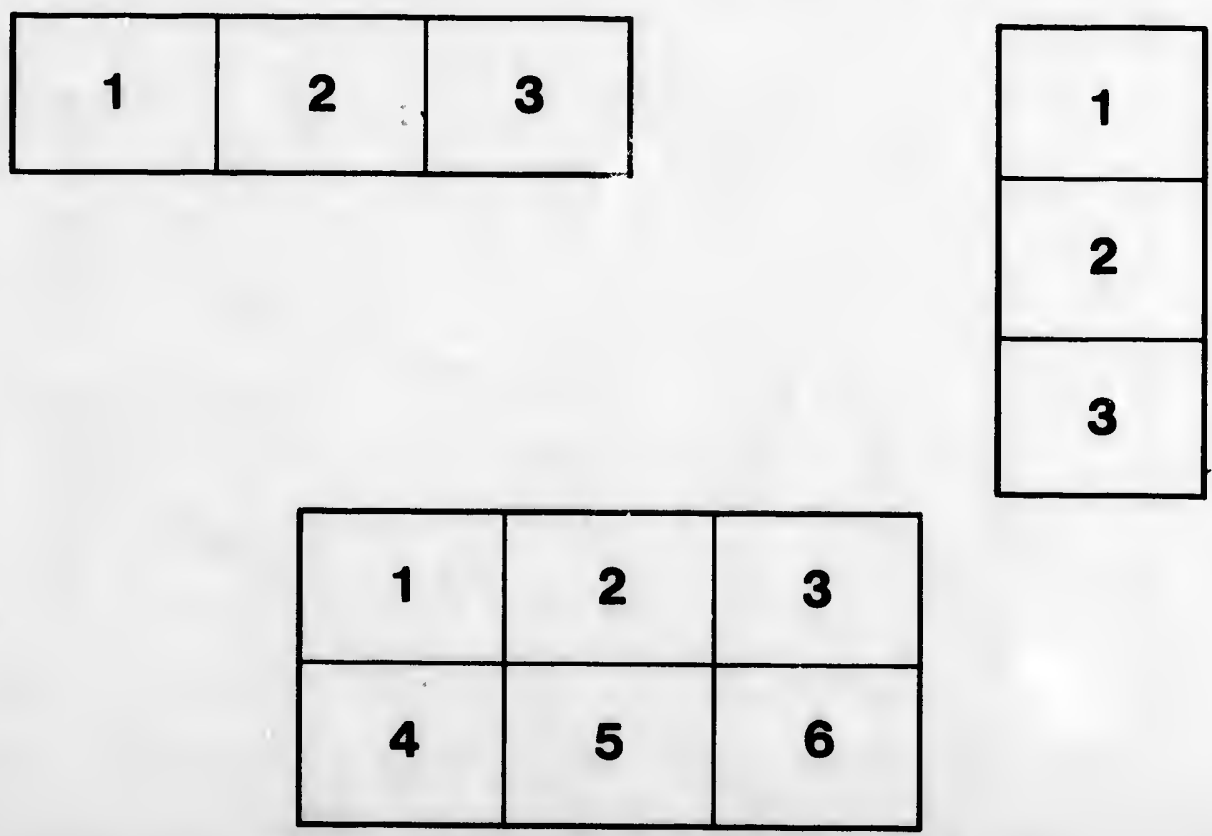


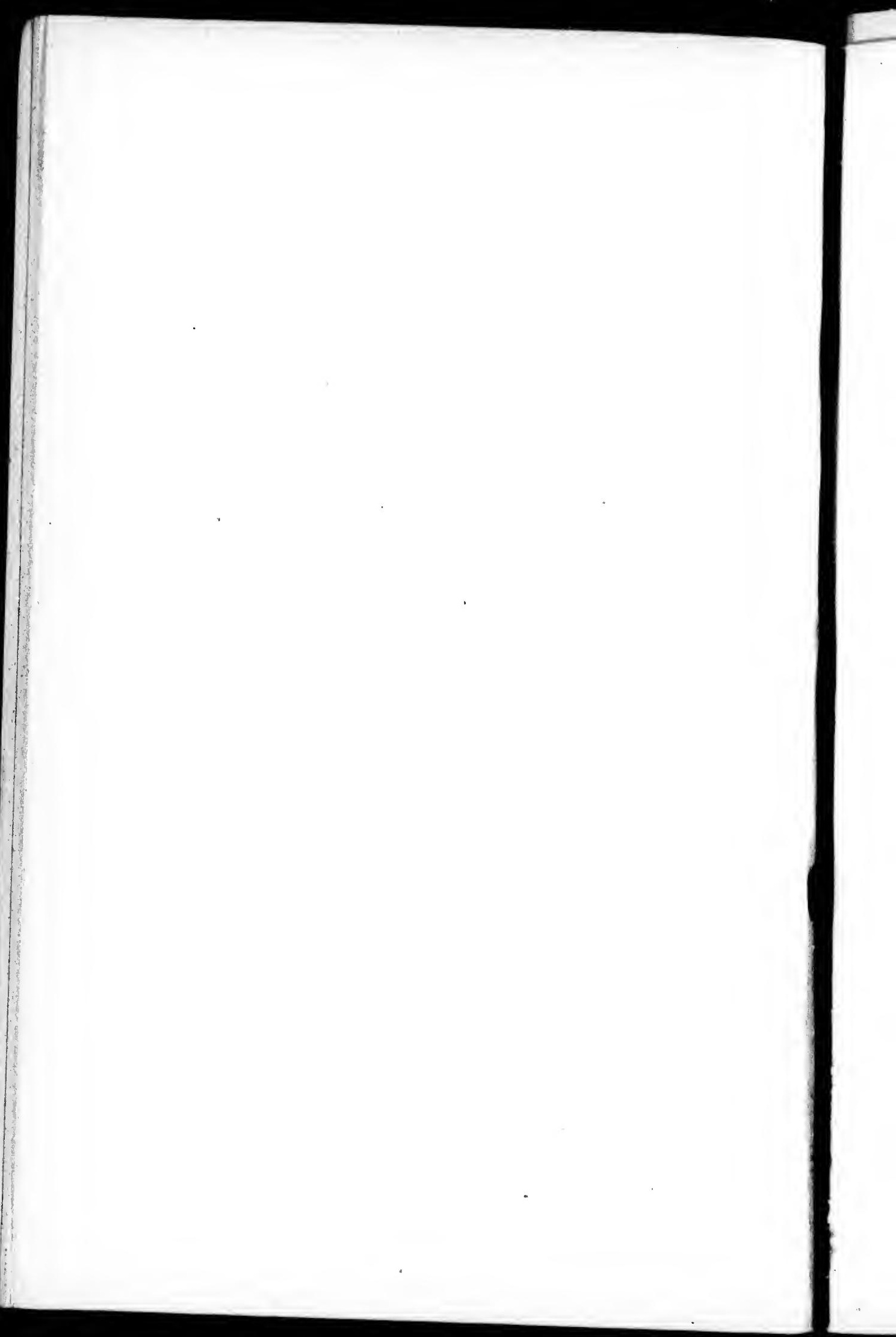




\section{LES AFFAIRES DE TERRE-NEUVE'.}

La question de Terre-Neuve est une des plus anciennes qui aient préoccupé la diplomatie européenne. Elle remonte à la découverte mèmẹ de l'île; dès le premier jour, Français et Anglais se sont trouvćs en présence et en rivalité. C'est une vieille histoire que ce conflit, mais une vieille histoire très actuelle.

Elle est actuelle parce que, ehaque annėe, 10,000 pècheurs de nos côtes vont chercher sur le French-Shore et sur les Grands-Bancs leurs moyens d'existence, parce que nos Iroits n'ont jamais été contestés avec autant d'âpreté, enfin parce que des négociations pénibles se sont poursuivies entre Paris et Londres, il y a peu de jours, pour arriver à la conelusion d'un accord lionorable sauvegardant tous les intérêts. Le malheur est que les intérèts en présence sont contradictoires et le moyen de les eoncilier semble aussi difficile à découvrir que la solution du problème de la quadrature du cercle.

Quoi qu'il en soit, on trouve de tout dans ce débat plusieurs fois séculaire : des occupations de territoires à main armėe, des actes de piraterie et de violence, des traités, des lois, des règlements, des ordonnances, mais surtout des discussions de textes et des subtilitès te mots dignes des casuistes le plus retors. Il a fourni aux journaux une abondante copie quotidienne, il a mème donné ì de graves législateurs l'occasion d'ègayer les discussions parlementaires par des calembours de vaudeville.

Nous ferons d'abord un bref historique des rapports de la France et de l'Angleterre au sujet de Terre-Neuve, depuis la découverte de l'ile. Nous examinerons ensuite les points sur lesquels portent les difficultes actuelles.

1. Voir le Livre Jaune publié par le ministère des Alraires étrangères sons le titre : ". Maires de Terle-Neuve " et distribué aux Clıambres françises le i6 mars 1891 , en uchme temps que les membres du Parlement britani(juc recevaient un Blue-Book traitant cles mêmes matiè'es. Les deux gonvernements s'étaient mis t'accord sur la date de publication de ecs recueils de documents. f.f. dèpêche de 11 . Waddington, du 11 mars 1891. 
Pour embrasser la yuestion dans son ensemble et la suirre dans les phases diverses qu'elle a traversees, il convient d'envisager trois périodes successives :

1" La première, partant de 1496 , comprend les $\mathrm{xv}^{\circ}$ et $\mathrm{x} y \mathrm{I}^{\prime \prime}$ siècles, et prend fin au traité d'Utrecht $(1713)$. C'est une pèriode de fail. Elle peut elle-mème se subdiviser :

a. De la fin du $x^{* *}$ à la fin du $\mathrm{XVI}^{\prime \prime}$ siècle la rivalité est générale entre les Anglais et les Frangais, les Espagnols et les Portugais;

6. De la fin du $x_{1}{ }^{\mathrm{C}}$ siècle à 1713, la lutte se restreint, les Français et les Anglais se disputent seuls Terre-Neuve et ses pècheries.

20 De 1713 à 18ïi, s’étend ce qu'on pourrait appeler la période de droit. C'est entre ces deux dates' que sont signés tous les traités qui fixent la part de chacun.

$3^{\circ}$ De 18507 à 1891 , se poursuit une période de discussion où tout est remis en question.

Premiène périodli, 1496-1713. - Bien que la moins florissante et avancée, Terre-Neuve est la plus ancienne des colonies de peuplement de la Grande-Bretagne.

Elle fut découverte en 1496 par Jean et Sébastien Cabot. Ce prenier voyage d'exploration, puis ceux que firent dans la suite Sébastien Cabot seul, et, après lui, le Portugais Cortereal attirìrent l'attention de l'Europe. L'extraordinaire richesse en poissons que ces navigateurs signalaient dans les parages qu'ils avaient visités, décidèrent de nombreux marins à explorer les eaux terre-neuviennes.

Dans les premiers teınps, l'Angleterre est faiblement représentée. Pourtant le gouvernement britannique ne marchande pas les encouragements à ses sujets " autant dans l'intérêt du commerce extérieur, " dit un édit de 10̈63, pour accroitre le nombre des pêcheurs et des " marins, et les ressources des villes maritimes, que pour économiser "les vivres frais du royaume ${ }^{2}$ ".

1. En rẻalitè, le dernier truitè statuant sur 'Terre-Neuve est celui de 1815 , et e'est a cette date qu'il faudrait peut-être, et pour ếre rigoureusement exact, ar'rèter la seconde pèriode. Mais la convention de $18: 37$ a une haute importance parce que, si les Terre-Neuviens out refusé de voter les lois nécessaires pour la l'endre elleclive, elle n'en oblige pas moins le gouvernement de la Reine, quil'a signèe et ratitièe. Sous nous refusons ubsolument ì admettre la prétention du gouvernement britannique, qui naccorde à ce texte de valeur que quand il y trouve son intėrît.

2. Voir dans la Recu: frausaise du for avril 1891, une intèressante ètude historique de M. A. Salaignac el des reuseignements techniques I ries complets sur la pêclse de la morue. 
La France ne reste pas non plus inactive. Henri IV place la péche à la morue sous la protection spéciale de l'État et en l'ait, pour ainsi dirc, une industrie nationale.

Pendant la plus grande partie du Xv" siècle, la concurrence se lait autant à coups de hache d'ahordage qu'à coups de fllet. I'lusieur's lois les Anglais, lorsque la pèche n'a pas été sulfisamment boune et ¡ju'ils rencontrent isolés des navires plus faibles, courent sus à ces rivaux et complètent leur cargaison de morue par celle de leurs captures.

Bientôt les nations ibériques se retirent. Elles tournent ver's d'autres horizons leurs ambitions et leur activití. Les Anglais et les Français restent seuls en présence à Terre-Neuve.

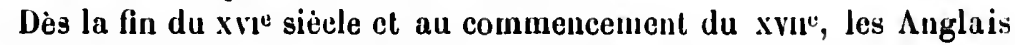
font des essais de colonisation. Le suceis ne couronne pas leur's entreprises. Quelques établissements se fondent pourtant par le fait de pècheurs qui, la saison linie, ne rentrent pas à leurs ports d'attache. Par contre le nombre des navires de pèche qu'envoie la cirandeBretagne augmente d année en année. En 1(il0, ils sont 200 montés par 10,000 hommes et le bénélice qu'ils retirent de leur industrie est évalué à 100,000 liv. st., chiffre énorme, surtout si l'on tient compte de l'époque et qui fait attribıer par lord Bacon aux Pècheries " une valeur plus grande que celle des mines lu Pérou ' ".

Én 1604, nos inarins avaient fonde Plaisance, puis en avaient fortilié la rade et, dès eetle époque, mais surtout le jour où Plaisance devient la résidence oflicielle d'un gouverneur français (1660), la lutte entre nos nationaux et les sujets britanniques prencl une tournure plus aiguë.

Pendant les guerres successives qui mettent aux prises l'Angleteric et la France, les Français saisissent chaque occasion de déloger les Anglais el de s'emparer des postes occupés par eux. Mais, chaque fois, les traités nous forcent ì évacuer les places conquiscs et la rivalité se perpètue.

En 1713, enfin, le traité d'Utrecht vient clore la période de lail pour inaugurer la période de droit.

Seconde íérode, 1713-1837. - L'article du traité de 1713 qui déliunite les positions relatives de la France el de l'Angleterre à 'l'errc-Neuvc est l'article 13. Il faut eiter in extensin ce document. Il est de la .plus grande importance : les traites et conventions qui l'ont suivi n'ont fait qu'en reproduire les termes, les contirmer et les paraphraser.

1. Revue frangraise, low. cil. 
Art. 13 du traité signé à Utrecht les 13 mars-11 avril 1713 entre la France el la Grande-Brelagne :

" L'ile de T'rere-Neuve avec les lles adjacentes appartiendront désormais à la Giande-Bretagne, el, il cette fin, le Roi Très-Clurétien feria remettre à ceux qui se trouveront à ce commis en ce pays-là, daus l'espace de sept mois i compter du jour de l'échange des ratifications de ce traité, ou plus tot, si laire se peut, la ville et le fort de Plaisance, et autres lieux que les Frangis pourraient encore posseder dans la dite ile, sans que le dit Roi Tris-Chrétien, ses héritiers ou successeurs ou quelques-uns de ses sujets, puissent desormais prétendre quoi que ce soit et en quelque temps que ce soit sur la dite ile et les iles adjacentes, en tout ou en partie. Il ne leur sera pas permis non plus 'd'y fortifier aucun lieu, ni d'y élablir aucune habitation en façon quelconque, si ce n'est des échalauds et cabanes nécessaires et usilés pour sécher le poisson, ni aborder dans la dite île dans d'autres temps que celıi qui est propre pour pècher et nćeessaire pour sicher le poisson. Dans la dite île il ne sera pas permis auxdits sujets de la France de pècher et de sécher le poisson en aucune partie que depuis le lieu appelé cap de Bona-Vista jusqu'à l'extrémitè septentrionale de la dite île, et de lá en suivant la partie occidentale jusqu'au lieu appelé l'ointe-Riche. "

Cie texte établissait au profit des Français un droit positif et un droit migulif: Un droit positif : la faculte de pêcher clans les eaux territoriales de l'ile et de se servir du rivage entre les points extrèmes ètablis; un droit négatif' : la garantie de n'ètre molestés par personne et sous aucun prétexte.

Ces dispositions du traite d'Utrecht ne pouvaient qu'entraver le développement normal de la population et de la prospérite de 'l'erreNeuve. C'est ce qui arriva. Mais les Anglais seraient assez mal venus à nous le reprocher, car ils ont maintenu longtemps le principe : " la cóte pour la pêche ", et les merchant-udvernlure's n'ont cessé de considèrer Terre-Neuve tant qu'a duré leur privilège comme " un grand " navire anglais ancré auprès des Bancs durant la saison de pèche, $\dot{a}$ "l'usage des marins a'Angleterre " ".

Apres 1713, la situation des colons s'amẹliora peu à peu. En 1729, le Board of Trade nomma un gouverneur, non toutefois sans soulever les protestations ènergiques des Fishing-ıdmirals ${ }^{2}$, qui voyaient désormais leur pouvoir contrôlé.

1. Revue fiuncaise, loc, cit.

$\therefore$. On noumait Fishing-tulmiral le premier capitaine arrivé qui devenait, sans 'ju'il eùt à remplir aucuue conditiou de capacité, juge suprếne dans le havre oı baie oì il s'était établi pour la saison. 
Pendant la guerre de Sepl-Ans, les Français firent, en s'emparant de Saint-Jean et en bombardant la cote anglaise, lcur dernière tentative de conquète territoriale.

Le traité de Paris (1763) fixe de nouveau, par son article $\ddot{~ ;, ~ l a ~ s i t u a . ~}$ tion et nous attribue les îles de Suint-Pierre et de Miquelon avec li restriction de ne pas les fortiller.

Cependant, la population de T'erre-Neuve augmente, lentement il est vrai. Ln 1763, elle est de 13,112 habitants; mais c'est une population pauvre et malheureuse, à la merci des armateurs anglais.

En 1764, le Labrador est adjuint à 'Terre-Seuve, pour permelloe aux

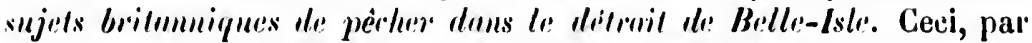
parenthise, prouve bien que nos droits sur le Fiench-shore etaient alors considérés comme exeiusifs.

En 177ว, le gouvernement de Georges III octroie aux navires ban'quicrs des primes semblables a celles qu'aujourd'hui on nous reproche tant.

Toutes les mesures préventives prises des deux parts n'empèchent pas les querelles de se renouveler sans cesse. Aussi, en 1783, au traité de Versailles, apris avoir maintenu " le roi de la Grande-Bretagne en la proprièté de T'erre-Neuve " ', change-t-on d'un commun accord les limites de la còte réservie aux Franẹais, pour éloigner ces derniers des centres anglais de la presqu'ile d'Avalon. Les points extrêmes désormais assignés au Fruch-Shore sont le cap Saint-Jean et le cap Raye ${ }^{2}$.

Mais le traité de 1783 n'était pas signé dans les mèmes conditions que celui de 1763. La France venait de prendre une belle revanche sur sa rivale. Elle avait brisé l'empire colonial de celle-ci et avait aidé ses plus riches colonies à se constituer en république indépendante. Au lieu d'imposer la paix, l'Angleterre la demandait. En France on s’étomna que M. de Vergennes, secrétaire du hoi pou les Affaires ètrangères, ne protitit pas de ces avantages pour éclamer les restilutions importantes. Il lut accusé de fuiblesse. La diplomatie de Versailles, alors, pour satisfaire dans une certaine mesure au mouvement de l'opinion publique, demanda au cabinet anglais que l'article ö du traité consacrit les droits exclusifs de la France dans la zone qui lui étail dévolue. Mais le ministère anglais craignait de vives attaques au Parlement. Il voulut éluder cette reconnaissance expresse et un moyen terme fut adoplé. Une déclaration el une contre-déclaration furent signées par les plénipotentiaires respectifs des deux puissances

1. Traite de Versailles, art. 4.

‥ Art. 3 . 
et jointes au corps du traité '. Seule la déclaration du roi Georges est intéressante pour notre thèse. En voici le texte :

..... "Pour que les peelieurs des deux nations ne fassent point naître de querelles journalières, Sa Majesté Britannique prendra les mesures les plus positives pour prévenir que ses sujets ne troublent en aucune unanière par leur concurrençe la pêche des Franțais pendant l'exereice temporaire qui leur est accorde sur les côtes de l'ile de Terre-Neuve; et elle fera retirer à cet eflet les élablissements séden. taires qui y seront lormés. Sa Majesté Britannique dounera des ordres pour que les pêcheurs français ne soient pas gênis dans la coupe de bois nécessaire pour la réparation de leurs échafuudages, cabanes et batiments de peche.

"L'art. 13 du traité d'Utrecht et la méthode de faire la péche qui a èté de tout temps reconnue seront le modèle sur lequel la péche s'y fera; on n'y contreviendra jas ni d'une part ni de l'autre..... "

"Ce sont là, remarque un publiciste canadien, pour tous les juges impartiaux des clauses bien claires, bien explicites, par lesquelles le roi d'Angleterre limitait sa souveraineté sur 'Terre-Neuve aussi formellement qu'avait pu le faire Louis $\mathbf{X I V}$, en 1713, fuand il s'engageait à détruire les fortilications et à combler le port de Dunkerque.

" Lin texte si précis, si catégorique, appuyé sur une jouissance incontestée et presque séculaire laisse peu de place à la discussion 2."

Ce témoignage d'un étranger désintéressẻ parait assez édifiant et utile à retenir.

Après le traité de 1783 le monopole des pècheur's français s'exerce sans contestation pendant une période de neuf ans et demi. Cette longue prise de possession mérite que l'attention s'y arrète. Les lirançais ont dès le dèbut interprété la clause que nous venons de rappeler dans le sens qu'ils lui donnent aujourd'hui, et il sernit pour le moins bien étrange que le gouvernement britannique n'eût pas èlevé d'objec. tion si un désaccord avait existé entre les deux diplomaties.

"Dès l'origine, les Français ont eu en leur faveur les conditions qui constituent le Plem!n! Dominium :

$1^{\circ}$ Un titre régulier;

$2^{\circ}$ Une entrée en possession avec longue jouissance sans eontestation $^{3}$. "

1. Voir dans Terre-Neuce et les Terre-Nenviens, par II. de I.a Chaume, l'analyse d'une brochure publièe en 1876 à Quèbec, en réponse á une autre brochurc contestaul nos droits, publiée à Londres par un avocat de la Nouvelle-L̈cosse. M. Witman.

2. Nêtue brochurc.

3. Loc. cil. 
Les traités intermédiaires entre 1783 et 181\% n'ont pas dimportance en la matière, ils n'npporlent aucun élément nouveau dans le lébat. En 181:, l'Angleterre voulait tirer de Waterloo un avantage matériel. Elle résolut d'enlever ì la France uno de ses dernières colonies et donnn le choix au gouvernement de Louis. .VIII entre les iles de Saint-Pierre et Miquelon avec les picheries de 'Terre-Nenve et l'ile de France (aujourd'hui Maurice). Ise duc de Hiclielieu opta pour les pécheries d'Amérique et le monopole de la Finnce l'ut restaurí, sur ce point, dans son intígrite.

Mais pendant les guerres de l'empire, les pecheurs français n'avnient pas fait nsage du Firmrh-Shore: les pécheurs terre-neuviens n'araient eu aucune concurrence à combatlre, les prix ılu poisson avaient triplé, des fortunes ènormes s'étaient élevées. Sur la còte ouest quelques villnges s'étaient foudés pendant notre absence et nous nous tronvions sur ce point en préscuce du fait aceompli.

Après le traité, la peche est reprise nvec vigueur et activité. Des primes sont necordées par la.France à ses marins, les prix baissent de plus de 2ij p. $0 / 0$ et une crise sciricuse se produit qui ruine plusieurs maisons de Saint-John.

Cependant Terre-Neuve, abandonnée pendant vingt ans à elleméme par les Anglais et les Français, avait pris son essor. La population, accrue dans de notables proportions, devenait exigeante. En 1825 , le gouvernement britannique cloit adjoindre un conseil au gouverneur; en 1832, Terre-Neuve obtient son parlement et en 18.54 le self government.

Débarrassée des Anglais. la colonie veut aussi évincer les Franı̧ais. Nos droits sont remis en question; on soutient qu'ils ne sont pas exclusifs.

Déjà en 1822, sir Charles Hamilton, gouverneur de l'ile, et en 1828 sir Th. John Cockiane, sou successeur, s'étaient vus dans l'obligation de rappeler en termes ènergicues leurs administrés au respect des traités '. Mais c'est en vain: les 'T'erre-Neuviens ne veulent pas entendre raison. En 18:3i, un certain $M$. Robinson se fait, au parlement d'Anglelerre, leur avocat. Il accusc le gouvernentent britannique de faiblesse, il lui reproche de sacrifier la colonie. Le ministère n'aceepte pas la discussion. Mais, en cette mème année 183:), comme les attaques se renouvellent, il se résout ì déférer la question de droit aux jurisconsultes de la couronne. Ils répondirent le 30 mai 18330 :

..... "Nous pensons que les sujets francais ont le droit exclusif de

1. Voir les proclamations de ces deux gouverneurs, limp .Imue, 1. 11, el discours de II. Bozérian nu Sénat, séance du li mai 1890. 
péchur sur la partie de la cóte de Terro-Nenve spécilléc dans le cinquième article du traitó définitif signei ì Versailles le il sep. tembre 1783.

Signé : Jolı Donson; J. Caмrnk:ı, ; H. W. HuLrk."

Les ministres ne voulurent pas alhêrer ir cette interprétntion. Ises juristes furent invités à examiner de nouveau les pièces et, le 17 avril 1837 , ils se ravisèrent, conformément au desir de leur gouvernement. Voici dans quels termes :

.... " Nous avons l'honneur de vous rendre comple que, nous reportant à l'opinion exprimée dans notre rappart dn :30 mai 18:35, nous pensons que nous avons été plus loin que ne le justillait la façon dout la question nous etnit posèe (than the circumstances of the case fucily "arrout).

"Attendu le traité de 1783 et la déclarntion qui lui est jointe ef l'acte dı Parleinent, nous pensons que la Grande-Bretagne a pris l'engngement de permettre aux sujets de la Firance de pêcher, pendant la saison, dans le district assigné, sans avoir ì suhir aucume interruption de la part des sujets britanniques.

"S'il existait réellement assez de place dans les limites du district en question pour que les pecheurs des deux nations pussent y pècher sans que des collisions dussent en résulter, nous ne peusons pas que la Grande--Bretagne serait astreinte à empecher ses sujets d'y pícher. Quoi qu'il en soit, il parait résulter du impport de l'amiral sir P. Ilalkett que c'est difficilement praticable; et nous sommes d'avis que, conformément à la véritable nature du Traité et de la Déclaration, il est interdit aux sujets britanniques de pecher s'ils causent quelque embarras à la pèche française.

Signé : J. Dodson; J. Campbell ; II. W. Rolfe '. "

En somme ce second avis n'infirme pas le premier. Il reconnaît que la péche commune ne peut pas se pratiquer et que par conséquent les Français doivent seuls exercer à Terre-Neuve eette industrie.

'Toutes ces attaques, cependant, conduisent le gouvernement français à considérer comme nécessaire une nouvelle confirmation de nos droits. Le gouvernement britannique reçoit sans enthousiasme les premières ouvertures qui lui sont faites. Il reconnait bien que " le privilège acquis aux pècheurs français a èté reconnu comme exelu-

1. Voir Livre Juune, p. 184; voir la dépèche de l'amiral Krantz à ll. lioblel, :1 septembre 1888. 
dans le

i) sep.

li.

ion. Less

17 avril

nement.

e, nolls

ai 18:3i;,

a fng̣on

the case.

ointe et

n pris

$r$, pen-

nucune

district

pecher

pas que

picher.

Halkett

, con-

, il est

uelque

)

onnaît

consé-

indus-

fran-

le nos

de les

"le

xelu-

ioblel,

sil', en prutique "', mais il recule devnnt une déclaration formello, qui pour'rnit lui erier de graves difficultés intérieures.

Le gouveruement l'rançais mnintient sa demande et, en 1857, alors que les deux gouvernements de Jondres et de Pniris sont unis d'une ètroite amitié, une convention est discutée et acceptée par les plénipotentiaires des deux puys. L'article $1^{\text {"N }}$ nous donnait pleiue sutisfaction : "Les sujets français nuront le droit exclusif de pecher et de so servir du rivage pour les besoins de leur péche. "

Sur la côte ouest, oì parmi les colons illégalement élablis se tronvaient nombre de Canadiens français, nous consentions à abandonnev certains points.

$\Lambda$ la nouvelle de cet arrangement une véritable sédlition éclate i 'Terre-Neuve. L'écusson royal est trainé dans la boue, les armes de ln reine sont attachíes a la queue d'un cheval et promenées ainsi par les rues de Saint-John. La législature de l'ile refuse son concours i l'exécution de l'nrrnngement signé.

La convention de 1857 n'ẹ a pas moins une importance extrêmement considérable. "Nalgré son avortement elle reste un document précieux; car elle témoigne que l'Angleterre, par l'orgnne de son gouvernement et de ses négociateurs officiels, a reconnu comme fondées les prétentions de la France. Cet épisode a mis la diplomatie britannique dans une situation embarrnssante, car l'absence de In signature royale ${ }^{2}$ n'annule pas les procès-verbaux des séances oì les plénipolentiaires anglais ont reconnu le régime créé depuis 7i. ans par la France ${ }^{3}$ "

Troisı̇̀.se périone, 1857-18\%1. - Ia troisième et dernière période à laquelle nous arrivons est la plus intéressante à tous égards.

La populution de 'Terre-Neuve, qui ètait de 121,000 habitants en 18507 , augmente rapidement. Elle alteint en 1869 146,000 hunes et 200,000 en 1890. Au fond la difficulté vient des modifications profondes que le cours des années a introduites dans la situation a Terre-Neuve depuis 1713, époque à laquelle le Frenrh-Shorr était désert. Avec l'augmentation de la population, la pèche est devenue moins rémunératrice. les insulaires ont dú chercher d'autres moỵens d'cxistence. Le temps était

1. Lord Palmerston, 10 juillet 1838.

2. Ceci est inexaet. La convention fut signée et ralifièe. Elle na pas ité mise i exėeution parce que l'assemblée législative de Terre-ícuve a refusé de voter les bills nècessaires pour la rendre effeetive. - Cf. Livre Jaune de 1\$91, 3, 13, nole 1.

3. Brochure cilée. 
passé oì, pour suffire à leur miscirable vie, ils vendaient l'appàt à nos marins, les aidaient à couper les bois nécessaires à leur industrie, garlaient leurs cabanes entre deux saisons. Les anciens habitants simplement tolérés avaient fait place à une population active, intelligente, voulant vivre. On explore l'intérieur de l'ìle, difficile à pénétrer à cause des forêls et des tourbières. On y trouve des vallées fertiles, de beaux bois, du charbon, des mines de cuivre. Mais toutes ces richesses sont inexploitables : la cote est fermée, partout les TerreNeuviens se heurtent à nos droits. En 187:, le gouvernement anglais fait cesser les travaux d'une mine et refuse d'autoriser la construction d'une voie ferrie transinsulaire aboutissant à la baie de Saint-Georges, parce que ses nationaux, pas plus que les noitres, ne peuvent avoir d'établissements permanents sur le French-shore.

Les Terre-Neuviens s'exaspèrent de la situation qui leur est faite. lls veulent se débarrasser de nous et nous cherchent des querelles de tous genres. Nous allons examiner tous leurs gricfs, passer successivement on revue les questions pendantes.

1. Las aroits de lic Finuer sunt-ils uxclusifs" - Lord Salisbury le conteste dans une note du 21 aoìt $188 i$ et dans une dépèche du !) juillet 1886. Pour soutenir cette thèse, le cabinet de Saint-James a fait remettre au gouvernement français un long memorandum à cette late du 9 juillet 1887 '.

Voici les élèments essentiels de son argumentation.

"Le Iroit de la France ne peut être une partie de son ancienne souveraineté qui lui est restée ", ear l'Angleterre a toujours étẻ souveraine de l'ile. Ce droit ne peut done plus se baser que sur les traités. Or le traité d'Ltrecht porte : "Il sera permis, ete. ", ceci est une simple concession de l'Angleterre. Dans le traité de Paris, de 1763, on lit : "L.es Francais ammel la liberté, etc. ", et cela n'est pas un droit non plus, mais ine autorisation.

lin 1782, II. de Vergennes anrait f'ait des ouvertures pour séparer les pècheries des Anglais de celles des Français afin d'éviter les querelles. I.e gouvernement anglais aurait relusé de reconnaitie les droits exclusils auxquels les Jirançais prétendent et le due de Manchesier avait ordre, au cas oi il ne pourrait obtenir le retrait du mot exclusif, l'opposer ì la conlue-déclaration française une nouvelle déclaration du roi d'Angleterre, aux termes de laquelle il entendait, par la 
J. GRUGhON. - LES AFFaIRES DE TERRE-NeUVE.

pât à nos strie, garits simpletelligente, énétrer à ertiles, de outes ces les Terreat anglais nstruction -Georges, 'ent avoir'

est faite. erelles de - successi-

lisbury le ípèclıe du t-James a Im à cette

ancienne étè souvees traités. ne simple i3, on lit : droit non

Ir séparer $r$ les queles droils anchesier ol exclue déclaraait, par la

concession de la pêche exclusive, ne s'engager à rien plus qu'à ordonner it ses sujets de ne pus molester pur leur concurrence, etc.

Aux négociations de 1801-1802, le gouvernement britannique refuse toute nouvelle concession parce que cela donnerait lieu à de " fort violentes clameurs et aurait des conséquences très fächeuses 1 ".

Une note de lord Palmerston de 1838 refuse énergiquement aux Français tout droit exclusif de pèche fondè sur un traité ou pièce justificalive quelconque?

L'avis des jurisconsultes de la couronne, rendu en 18:3̈, est modifiẻ en 1837, "apries un examen plus approfondi " .

Quant à la note de $M$. Labouchère en date du 16 janvier 183̆ 7 , " toute son argumentation est fondée sur la mème considération. savoir : que les droits des Français soient, en stricte logique, exclusifs ou non, ils le sont dans la pratique. Mais ce serait lá une question de fait et il convient de rappeler que la depiche de M. Labouchère a été éerite en vue de recommander à la colonie d'adhérer à la convention de 185̆." "

En définitive, lord Salisbury estime qu’il " $y$ a place pour les pècheurs des deux nations, pourvu que les précautions voulues soient prises ".

Telle est l'argumentation anglaise. Qu'avons nous à y opposer?

L'article 13 du traité d'Jitrecht ne dit pas que les Anglais ne pourront point s'établir sur le French-Shore et cependant les hommes d'État d'outre-Manche n'ont jamais contesté notre droit de faire expulser leurs nationaux. S’ils avaient eu le droit de pècher concurremınent avec nous, les Anglais auraient pu vivre à nos còtés; or toutes les conventions y sont contraires. Celle de 1788 notamment porte que le roi Georges " menulra les mesures lis plus positives " pour èviter ce contact.

Jusqu'en 1831 aucun doute ne paraît s'ètre élevé. Jamais le gouvernement britannique n'eut l'idée de discuter nos droits. Il fallut tout le bruit fait par les Terre-Neuviens et les discours de M. Robinso ॥ pour qu'il songeàt à consulter ses juristes. Entré dans cette voie, il est plus qu'évident que la réponse le 183ä n'était pas faite pour lui

1. Dèpêche de lord Cornwallis, 26 novembre $1 S 0 \mathrm{t}$.

2. Nous soulignons ce dernier membre de plirase parce yue celte " pièe justilicative quelconque " nous la trouvons dans la convention de $18: \%$. Ce texte est tout a fait positif; au surplus, et si l'on vent autre chose. en nous donnant i choisir entre Maurice et les l'icheries, l'Angleterre ne nous a-l-elle pas reconnu la proprièté exclusive du droit de péche à Terre-Neuve comme nous lui cédions et reconnaissions la propriète territoriale exclusive de lile de France?

3. On a vu plus haut que cet " examen plus approfondi " n'a pas été prècisément spontané de la part des jurisconsultes de la couronne.

A. Tome VI. -1891 . 
plaire. Elle était par trop catégorique; et l'invitation qu'il adresse aux jurisconsultes de la couronne de procéder ì un nouvel examen plus approfondi de la question, est un délicieux euphémisme.

Mais ce qui nous semble capital, dans ce débat, c'est la convention de 18.7. Les Terre-Neuviens n'ont pas voulu en entendre parler, malgré l'èloquence de M. Labouchère, et nous comprenons fort bien le sentiment des Terre-Neuviens. Ce que nous ne comprenons pas du tout, cest le peu de souci que le cabinel de Saint-James parait prendre de sa réputation de bonne foi, en répudiant les déclarations des plinipotentiaires anglais appuyées de la signature et du sceau royaux. Tout cela n'est pas digne viaiment du gouvernement d'une grande nation. Et, à l'heure actuelle, il semble bien qu'en Angleterre l'opinion publique s'en rende compte. On revient à une appréciation plus saine de la question et un grand journal de Londres n'hésite pas à declarer que "les droits de la France sont incontestables " 2 . Mème il n'éprouve pas de scrupules à ajouter qu'ils sont incontestés.

A la question des droils exclusifs se lie étroitement celle des villages du French-Shore. Ils se sont fondés par surprise pendant les guerres lu premier cmpire et aujourd'hui nous nous trouvons en présence du fait acquis. Jusqu'à ces dernières années, ces villages n'avaient élé que tolèrés; mais, en y nommant des magistrats, le gouvernement anglais a reconnu offieiellement leur existence et, par ce fait, manqué à l'exécution des traités.

2. Les Homurderies. Poissons el Cruslacis. - Depuis quelques années des Français ont fondé à 'Terre-Neuve des homarderies. Et cette industrie a pris une extension considérable lorsquaprès la vote du bail-bill " on essaya de tirer parti du homard comme appât ". Lit première homarderie date de 1886. Ic gouvernement britannique protesta sans retard, sous prétexte qu'il s'agissait de " bâtiments permanents ". La réclamation était fordée, le gouvernement français n'hésita pas à lui faire accueil : on supprima les constructions permanentes. Mais ce n'ètait point ce qu'on voulait à Terre-Neuve et l'on ne mit pas longtemps à trouver d'autres sujets de querelle.

On prétendit que non seulement nous ne pouvions construire des établissements à poste fixe. mais encore que les Français n'ètaient point autorisés à pécher le homard. Et cela parce que. " si les auteurs des traités, dit un journal de Saint-John, avaient voulu donner aux Français le droit de pècher autre chose que la morue,

1. Daily Noure, i janvier 1891.

2. Voir infin, p. 193 .

3. Voir discours de .1I. Flonrens, i la chambre des députés, séance du 20 janvier 1890. 
alors, sans doute, ils auraient employé le mot poisson et non le mot mmine " '.

Une paraille argumentation parait véritablement indigne de toute attentirn irtout lorsque les textes sont transfigurés avec une mauvaise foi i ussi évidente. Nous ne nous arrèterions done pas à la question de savoir si le homard est un poisson, si le Premier ministre de la Reine n'avait ramassé l'argument. Mais lord Salisbury a jugé convenable de se faire l'écho des facétieux diplomates en chambre de 'Terre-Neuve et dans une dépêche dı 28 mars 1889 à notre ambassadeur à Londres, il écrit textuellement : "Sur la question de savoir si les crustacés sont des poissons, les deux gonvemements sont divisés d'opinion."

Nous n'essaierons pas de trancher la question : il doit falloir pour la discuter avec le gouvernement de la keine être un très distingué naturaliste. Mais, ainsi que l'a remarqué $M$. Bozérian ${ }^{2}$, ne dit-on pas : "la pèche aux écrevisses " "? Au surplus on peut se demander si les plénipotentiaires de 1713 avaient fait de la zoologie aquatique et de la pisciculture une étude approforidie.

Le Premier ministre anglais sent bien que son argument manque de poids. Il en cherche un autre aussitòt et le trouve dans cette disposition de la déclaration de 1783 : "Le mode suivant lequel la pèche aura lieu ne pourra ìtre changé par aucune des deux parties, les pècheurs français ne bàtissant rien que leur's échafaudages ".... Or l'industrie nouvelle nécessite des ateliers spéciaux, et le gouverucment britannique en prend acte pour protester contre des opérations qui n'avaient pas èté prévues. Mais si nous n'avons pas le droit de pêeher le homard, les Anglais n'en ont pas le droit davantage, puisque " aucune des deux parties ne pourra changer le mode de pèche".

En réalité, les traités ne pouvaient pas pałler de la pêche du homard, parce qu'en 1713 nul n'y songeait, pas plus d'ailleurs qu'à la pêche dı saumon à laquelle pourtant personne ne s'est opposé. Ėı nous réservant le droit de pêche sur le Frrmilh-Shore nous avons entendu y comprendre toms les animaud qui vivent dans l'enu.

Et en résumé nous avons le droit de pêcher le homard :

$1^{\circ}$ Parce que les traités, entendus de bonne foi, nous garantissent sur le French-shore un droit de pèche sans restriction, ainsi que l'usage de la côte pour les produits;

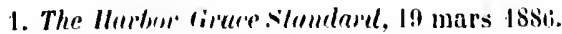

2. Discours au Sénat, 10 mai 1890.

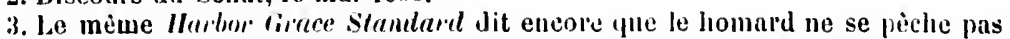
(to fish), mais se capture, sattrape (to catch). Ce qui ne i'empèche pas d'intiluler son article : " Lobster-flshery ", La pêche du homard. 
2" Parce qu'on ne pourrait concevoir un droit concurrent ou privilégié pour les Anglais de se livrer à la mème industrie qu'à la condition d'admettre, d'une part, qu'ils peuvent, là où ils se trouvent, nous empêcher de capturer la morue, ce qui reviendrait ì dire que les traités sont sans valeur, et d'autre part qu'ils ont la faculté de s’établir sur la cóte, ce qui est implicitement interdit par la déclaration du roi Georges '

3. Ouestion de's primes. - La mesure prise par le gouvernement français d'accorder des primes à la grande pêche a vivement irriti les T'erre-Neuviens et peut être considérée comme une des plus fortes raisons qu'ils font valoir aupris du gouvernement de la lieine pour continuer la lulte contre nous. Cette question a fait l'objet d'un échange intéressant de notes entre les cabinets de Londres et de Paris.

- Les primes accordées par le gouvernement français atteignent prìs de 50 p. (1)/0 de la valeur de la marchandise. La morue introduite en France ne paie pas de droits, et nos pècheurs ont à leur disposition des entrepôts extrènement favorables, comme Bordeaux, d'où il leur est aisé de l'exporter. Ces avantages l'aits à nos nationaux mettent les pêcheurs terre-neuviens dans la presque impossibilité de leur faire une concurrence utile.

Mais le gouvernement britannique ne doit pas oublier qu'il a longtemps luì-mème pratiqué le système des primes. Au surplus, ainsi que l'a remarqué Sir James Fergusson, à la chambre des Communes, c'est là un fait d'administration intérieure où le cabinet anglais ne saurait valablement intervenir. Les Terre-Neuviens paraissent l'avoir compris et, à en croire les journaux, ils demanderaient maintenant l'établissement d'un systime de primes à accorder par la GrandeBretagne aux exportations terre-neuviennes de morue. Ces primes Equivaugdraient aux primes françaises et perınettraient aux pècheurs insulaires de soutenir la concurrence.

4. Lı Boëllי. - İn somme, les difficultés n'étaient pas insurmontables. En 188:i, après neuf ans de pourparlers, les deux gouvernements arrivèrent à conclure un arrangement 9 qui maintenait formellement nos droits. Mais la lègislature de Terre-Neuve ne voulut pas entendre raison. Elle refusa de voter les lois qui devaient assurer la mise en pratique de la convention.

Le 1/4 novembre 188ö, un nouvel accord intervint qui annulait le précédent. La disposition de l'article II de l'arrangement de 1881, par

1. Cf. nole de l'amiral liranty, 30 janvier 1889.

2. Ice jij arril 188'. 
laquelle il était stipulé qu'aucune nouvelle construction anglaise ne serait ètablie sur le rivage de l'ìle dans les limites mentionnées, était maintenue; mais, pour donner satisfaction aux plaintes de la colonie, le nouvel article III prévoyait le cas où une mine serait déconverte dans le voisinage du littoral et réglementait le mode d'exploitation qui devrait être employè, de manière à " ne pas entraver le libre exercice de la pèche française ".

Une fois encore les Terre-Neuviens refusent de faire honneur aux engagements de la métropole. Ils basent leur refus sur deux articles qu'en 1884 ils avaient laissé passer sans protestation.

L'article XVI (ancien art. XV) exemptait de toute taxe les pècheurs français " pour l'introduction dans la partie de l'ile de Terre-Neuve comprise entre le cap Saint-Jean et le cap Haye en passant par le nord, de tous objets, matières, vivres, etc., nécessaires à leur subsistance et à leur ètablissement temporaire sur la côte de cette possession britannique? " Or il paraitrait que quelques capitaines ont abusé de ces franchises pour se livrer it un commerce frauduleux de spiritueux qu'ils faisaient pénétrer dans l'ile sans acquitter de droits de douane. Le patron d'un navire français, lı Glımeuse, serait même allè jusqu'à prendre le large ayant à son bord deux douaniers anglais venus pour procéder à des constatations légales. Cet incident fit l'objet d'un échange de notes dans le courant de février 1886.

Mais ce n'ètait là en somme qu'une question de mince importance. Le gouvernement français promit de prendre les mesures que le cas comportait.

La grande pierre d'achoppement fut l'article XVII (ancien art. XVI), qui reconnaissait aux pècheurs franẹais " le droit d'acheter la boëtte, hareng et capalan, à terre ou à la mer, Jans les parages de Terre-Neuve, sans droits ni entraves quelconques, postérieurement au cinquième jour d'avril de chaque année jusqu'à la fin de la saison de peche. "Les Terre-Neuviens voulaient ruiner notre industrie. Ils pensèrent en avoir trouvé le moyen en privant nos pècheurs de l'appât indispensable. Dans ce but fut soumis au parlement local un bill qui visait directement l'article XVII de la convention. M. Pennell, un des négociateurs anglais de l'arrangement, envoyè à Terre-Neuve pour en faire accepter les dispositions, échoua, et malgré ses efforts, le 18 mai 1886, le Bait-Bill fut voté. Il prohibait la vente de l'appit aux pécheurs étrangers à partir du 31 décembre 1887.

Le gouvernement français, en présence de l'attitude de TerreNeuve, prit immeddiatement une position très ferme. Le 21 juin 1881 , I. Waddington avisa lord Rosebery, secrétaire d'ĺtat pour le Foreign-Office, que le gouvernement de la République avait pris la 
résolution " d'exercer dans toute leur étendue et dans toute leur rigueur les droits que nous tenons des traités ".

"Lal diplomatie britannique n'a pas de scrupules à la légère ' ", elle en eut cette fois ; aussi bien, peut-ètre, furent-ils éveillès par l'attitude du ministere français. En mars et juin 1886, lord Granville et lord Hosebery donnent les assurances les plus formelles, touchant le maintien du libre trafic de la boütte. Mais bientôt des bruits fìcheux cireulent, et lord Salisbury, le nouveau principal secrètaire d'État pour les affaires ètrangères, déclarc à M. Waddington que a la pècherie anglaise de 'Terre-Neuve est écraséc par notre système de primes el qu'à son avis la libre vente de la boütte est une question sur laquelle on pcut discuter ${ }^{2}$ ". Notre Département des affaires étrangères proteste contre cette théorie et obtient que rien ne scra changé pour la campagne de péche imminente. Après un second vote du bill par le parlement de Terre-Neuve, le gouvernement de la Reine sanctioune enfin cet acte législatif qui ne deviendra applicable qu'en 1888.

Immédiatement, les pècheurs français se mirent en mesure de se fournir eux-mèmes de boütte, et en 1888, tous nos navircs purent facilement s'approvisionner dans la baie de Saint-Georges. Mais e'ètait une perte de temps pour les pècheurs des Grands-Bancs; elle fut évitẻe l'année suivante par l'emploi de boëttes conservèes ct d'un coquillage nommé bulol ou coucou qui se trouve en grande abondance sur les bancs mèmes. L'essai pratiqué a donné les meilleurs résultats et le Bait-Bill, dirigè contre nous, n'a réussi qu'à priver 'T'erre-Neuve de plus d'un million de franes que nos pècheurs y laissaient chaque année en échange de la boëtte. En outre, le maigre budget de l'ile est grevé d'une lourde charge pour la surveillance des côtes.

On voit done que le but poursuivi par le parlement terre-neuvien a été absolument manqué. C'est ce qu'a reconnu sir W. Whiteway ", le premier ministre actuel de la colonie : "Les grandes dépenses qu'exige l'exécution du Bait-Bill, a-t-il dit, ne sont nullement compensées par les avantages qui en résultent; il est nécessaire d'adopter d'antres mesures pour assurer effectivement la protection et la conservation de nos intérêts quant aux pecheries. "

5. Lu question des truppes. - On nomme trappes à morue une solte de grands filets qui constiluent de véritables barrages, arrêtent la morue et l'empêchent de pénètrer dans les baies. Le gouvernement français en avait permis l'emploi à ses ressortissants depuis 188ュ, mais on a reconnu que ces engins sont absolument nuisibles et

1. M. Flourens, discours citè.

2. Wépèche de M. Waddington, 21 janvier $188 \%$.

3. Dans un manifeste électoral. 
destructeurs et nos armateurs eux-mèmes ont été les premiers à en réclamer unanimement la suppression. Les trappes l'urent interdites en 1888. Dernitrement les Terre-Neuviens ont voulu les rétablir, mais le gouvernement métropolitain s'y est énergiquement relusé.

\section{III}

Toutes ces questions ont fait l'objet de longues et pénibles négociations, rendues plus irritantes par des incilents continuels. Tantòt e'est le cas de lı Cilmmeuse que nous avons rappelé plus haut, tantôt c'est un autre capitaine franegais qui saisit un filet terre-neuvien et prend la mer, emmenant ì son bord l'huissier qui vient lui signifier d'avoir à comparaitre devant un tribunal local.

De leur côté les pêcheurs français se plaignent continuellement des vexitions dont ils ont à soulfrir. Un jour e'est le patron du schooner britannique le Dar' qui s'empare d'un filet francais ${ }^{1}$; une autie fois ee sont des armateurs eomme MM. Besnier et Dupuis-Robial qui font des réclamations au sujet des préjudices que leur causent des pêcheurs anglais ". Dans chacun de ses rapports, le chef de notre mission navale signale les actes répréhensibles que commettent sur les còtes du Frrmilh-S/hmi! les goélettes nomades qui chaque année quittent les baies du sud pour se rendre au Labrador et profitent de leurs reliches dans les havres oceupés par les pècheurs français, pour se livrer í toutes sortes de déprédations. Les attentats de ces maraudeurs sont si fréquents qu'on les nomme Wrecliers (naufrageurs, pillards de mer) ${ }^{3}$.

Dès 1882, on songe à adopter un mulus vivemuli provisoire. L'idée est reprise en 1889 et le 11 mar's 1890 elle aboutit.

Les questions de principe et les droits respectifs ètant entièrement réservés, on convient du maintien clu stıtu quo pour la saison qui va s'ouvrir. Aucune homarderie ne fonetionnant pas antérieurement au $1^{\text {ar }}$ juillet 1889 ne sera admise, à moins que les commandants des deux stations navales n'en tombent simultanément d'accord.

Bien que tout à fait provisoire, ce modus vicendi ne salisfait personne. En France, il est attaqué par la presse, et M. Bozérian déclare au Sénat 'qu'il ne saurait en penser beaucoup de bien. A 'TerreNeuve, voiei comment le traite, dans un meeting, sir James Winter :

1. Lirre Janne de 1891, p. 69 ì 73.

2. Ilid., p. 133, 13̈̈, 1:35 et 179.

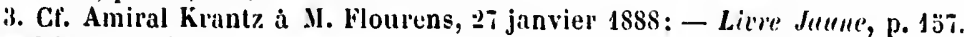

4. Discours cité. 
"Qu'est-ce que signifie ee morlus vimmdi?

" Un voleur entre chez vous et s'empare de votre vaisselle. Vous le menacez de le livrer à la justice et il vous propose un modus vivendi aux termes duquel il gardera la vaisselle pendant trois mois tout en rèservant vos droits de propriété absolue sur elle, mais sans que vous-mème puissiez dès lors vous en servir. Accepteriez-rous ce modus virmeli? Non sans doute, vous livreriez le coquin aux juges. Eh bien! ce modıs vicendi arrèlè entre l'Augleterre et la France n'est pas autre chose que le modus virendi imagine tout à l'heure pour le vol de la vaisselle. "Le Mrily Chronicle qui reproduit ce joli discours ajoute : " La comparaison a de la forec. "Nous ne ferons, pour notre part, qu'une simple réflexion : Sir. J. Winter oublie de dire qui, de 'Terre-Neuve ou de nous, est le propriètaire lésé, et qui, le voleur de vaisselle. Peut-ètre le lecteur ne conclura-t-il pas selon les désirs le l'orateur d'outre-mer'.

Cet aceord provisoire tant critiqué a lommé cependant de bous résultats. Grice à lui nos marins n’ont pas été troublés dans leurs opérations. A Brig-Bay el ì Sainte-Marguerite, oú nous avions eu les plus grandes difficultés en 1889, les pècheurs anglais et français se sont mis eux-mèmes d'accord sans attendre l'intervention officielle '.

En mème temps qu'on essayait de dètendre lit situation par l'adoption l'un modus vivendi provisoire, on songeait à la régler définitivement par voie d'arbitrage, puisque les négociations directes ne pouvaient pas aboutir. En avril 1890 , le gouvernement de la Reine manifestait le dèsir de reprendre la question dans son ensemble; en mai, lord Salisbury propose officieusement de recourir à l'arbitrage. M. Waddington réplique aussitôt qu'un arbitrage sur l'ensemble, de la question impliquerait que les droits de la France sont douteux; que tout au plus on en pourrait concevoir l'application it un point non visé spécialement par les traités, les homarderies par exemple; qu'aı surplus il est sans instructions.

Lc gouvernement français fait bon accueil à la proposition d'arbitrage limité qui lui est transmise. Les points litigieux sur lesquels l'arbitre devra .. prononcer sont mis en discussion. A la suite d'un échange de vues en février 1891, le Foreign-Office et le ministère français des Affaires ètrangères tombent d'aceord pour dẻcider qu'une commission arbitrale sera nommée. Ln arrangement dans ce sens est signé à Londres le 11 mars 1891. Ies dispositions importantes le cette convention sont les suivantes :

1. Cr. Rapport dı capitaine de vaisseau Maréclıal, commandant la station navale de Terre-Neuve, an ministre de la marine, 11 vetobre 1890. 
$I^{\circ}$ La commission arbitrale jugera et tranchera toutes les questions te principe qui lui seront soumises par l'un ou l'autre gouvernement concernant la péche du homard et sa préparation.

20 Les deux gouvernements s'engagent à exícuter les décisions de la commission arbitrale.

$3^{\circ}$ Le modus vivendi de 1890 est renouvelé pour $(89) 1$.

$4^{\circ}$ Quand elle aura tranché la question des homarderies, la commission arbitrale pourra être saisie d'autres questions subsidiaires relatives aux pécheries de Terre-Neuve et sur le texte desquelles les deux gouvernements seront prẻalablement tombés d'accord.

כ0 La commission arbitrale sera composée de trois spécialistes ou jurisconsultes désignés du consentement commun des deux gouvernements et de deux déléguiss de ehaque pays qui seront les intermé. diaires autorisés entre leurs gouvernements et les autres arbitres ${ }^{1}$.

Une seule question va done èlre résolue, celle des homarderies. C"est la plus urgente à tous égards, surtout parce que si l'on n'arrète pas des mesures énergiques les fonds de homards seront épuisés il'ici moins de deux ans ".

Lorsque la commission se seril prononcée sur ce premier point, il est possible, mais peu probable, qu'il lui en soit soumis d'autres. S'il en ètait ainsi, elle pourrait trancher successivement toutes les questions pendantes; mais il ne faut pas esperrer que le ccnflit prenne lin par cette voie.

L'opposition des 'Terre-Neuviens ne désarmera pas. Ce qu'ils veulent, c'est 'Terre-Neuve aux T'erre-Neuviens, et cela est impossible lant que nous serons là. Les conventions de 18507, 188., 188.; auraient seulement localisé le mal, ainsi que le remarque sir Charles Dilke ${ }^{3}$. Notre droil, qui emporte limitation de la souveraineté territoriale, constitue une anomalie à notre époque. Le patriotisme local des 'lerre-Neuviens, d'ailleur's parfaitement respectable, joint à tous les intérêts commerciaux et industricls de l'ile, souffre de la présence des Franeais. Si l'Angleterre ne sait pas obtenir notre éloignement. elle risque de provoquer des événements irréparables. Les Terre-

1. Les trois jurisconsultes désignés a'un commun accord sont MII. de Martens, professeur de droit des gens à l'université de saint-Pètersbourg; Rivier, consul général de Suisse à Bruxelles, président de l'Institut de Uroit international, et irann, ancien membre de la Cour suprème de Norvège.

2. Mapport de fin d'annċe du capitaine de vaisseau Marćchal, 1890.

3. Cf. Problems of Greater Brilain. 
Neuviens sont déeidés in se défendre par tous les. moyens. Leurs représentants les plus autorisés te déclareut hautement'. Ils ont parlè de faire appel aux autres colonies angluises. Ils ont nussi manifesté des vellèités de demander leur entrée dans l'Union américaine. Cie ne serait pas une solụtion. Il est diffleile de prévoir l'aeeueil que les litats-Unis feraient î pareil désir. Mais la réponse de la république américaine fitt-elle favorable, nos droits ne seraient en rien infirmès, car une servilude - et e'est bien un droit de servitude que nous possédons à Terre-Neuve - ne s'èteint pas par ce fiait que le fonds servant eliange de propriétaire. 'Tout ce que pourra faire la colonie n'est done dangereux que pour si métropole, et e'est a celle-ei d'aviser.

Ces considerations ont mene à envisager l'hypothèse d'une transaction. L'idẻe, émise en Angleterre, a été diseutèe des deux côtés de la Nanche. On s'est demandé quelles seraient les compensations possibles ì une cession éventuelle de nos droits. On en a proposé de nombreuses.

Certains journaux, te Daily-Ni'm's entre autres, ont prétendu que notre gouvernement aurait voulu traiter solidairement les deux questions d'Ígypte et de Terre-Neuve. On a parlé aussi de Maurice. On s'est rappelé qu'en 181:, M. de Riehelien avait dù choisir entre cette ile et les pécheries " et l'on a pensé qu'un éehange serait praticable. Une troisième proposition tendrait a faire ollirir par la Grande-Bretagne l'abandon des Nouvelles-Héhrides. Quelyues esprits mis en éveil par la cession d'IIeligoland i l'Allemagne voudraient voir Jersey. et Guernesey faire l'objet d'une cession analogue ì la France.

En dehors de ces diflërentes solutions, il nous reste encore deux propositions ì signaler.

La première est due it M. Paul Leroy-Beaulieu ".

La Tunisie est liée à la Grande-Bretagne par un traité de commeree, qui accorde à cette puissance le traitement de la nation la plus favorisè '. Ce traité n'a pas d'échéance lise et ne pourra prendre fin que du consentement mutuel des deux hautes parties contractantes. M. Leroy-Beaulieu pense que nos droits sur Terre-Neuve étant de nature plus ou moins analogue à la servitude commerciale dont le Bey a grevé la Régence au profit de l'Angleterre avant notre oceupation, on pourrait échanger l'un contre l'autre ces deux privilèges.

1. Voir dans le Temps du 2ö décembre 1890 , me inlerview de M. Robert Bond, secrétaire colonial de Terre. Neuve.

2. Voir swmer, p. $48 \%$.

3. Voir I'Economiste fromgrais du 21 juin 1890.

i. Il existe bien aussi un traité de conmerce italo-lunisien, mais il expire dans six ans et il sufli ra de ne pas le renouveler. 
Il faudrait seulement exiger en outre une indemnití pécuninire a distribuer ì nos pecheurs lésés.

Au moment où M. Paul Leroy-Benulieu suggérait eetle combinaison, les produits tunisiens étaient encore soumis, à leur importution en France, aux droits du tarif général, et, par suite des rísistances de certains intérèts régionuux, on liésitail ì leur necorder un traitement plus favorable sans stipuler, en retour, des avnntages pour les produits français importés en 'Tunisic. On avait été amené ningi ì songer' ì l'établissement d'une union douanière entre la France el la 'Tunisie. Mais l'existence du traité anglo-tunisien était un obstacle in l'établissement de cette union. Depuis lors, une loi, promulguće le I!) juillet 18!0), est venue modilier le tarif géuéral les douanes en faveur de certains produits tunisiens. Il n'est plus question d'union dounnière entre la France et la Tunisie. L'abandon du traité anglo-tunisien ne présente done plus autant d'intérè qu'umparavint '.

On a parlí entin d'une cession territoriale en (iambie. L'Angleterre possède sur cę point de la eóte africaino une grande enclave qui sípare notre colonie dı Sénégal. Dans un artiele récemment publisi par le Daily Crrophic, sir Ch. Dilke aftirme que le gonvernement de la République a repoussé l'ofire de la Gambie qu'il considere comme une compensation insuffisante.

Quoi qu'il en soit, nous n'avons pas ì formuler de voux. Le gouvernement britannique doit savoir mieux que personne combien la situation dans Iaquelle il se trouve est lélicate. "Depuis quelques années l'Angleterre ne semble gonverner ses eolonies qu'à la condition de leur obéir. "Si un pareil systeme lui convient, libre ì elle de elle de le conserver. Mais s'il pluit aux 'T'erie-Neuviens de violer nos droits, l'Angleterre ne saurail avoir la prétention de se lérober derrière le silf guvernmint de sa colonie. Nous n'avons pas ì discute $\mathbf{r}^{\prime}$ avec les ministres de Saint-John, nous ne connaissons que le cabinet anglais qui a signé les traités. C'est à lui qu'il appartient d'envisager les conjonctures et de chereher une combinaison qui le tire de l'impasse où il est engagć. Nos droits qui sont incontestables nous satisfont pleinement. Iui seul en est gèné; ce n'est done pas à nous de prendre l'initiative.

Mais il nous semble que si le gouvernement de la Reine donne des preuves sérieuses de bonne volonté, si le désir qu’il manifeste d'arriver à une entente n'est pas une fiction destince à gagner du temps par des négociations vaines, la France n'a nucune raison pour re-

1. Sur le régime donanier de la Tunisie. voir dans les Ammalss, annece 1884. p. file et suiv., l'article de N. P. d'Orgeval. 
pousser de prime nhord et de parti pris les oflres qui pourruient lui itro faites.

la firench-ithore nous est peut-ìtre, du moins pour le moment. moins indispensable qu'à l'époque oì l'on sichait la moł'ue. Aujourd'hui les navires-banquiers salent i bord beaucoup de morue et ln rapportent en liurope ninsi priparíe; c'est ce qu'on appelle la morue verte.

A la vérité, la possession llu frirurh-S/murt présente encore pour nons un sérienx intérìt. Ia grande péche est, il ne l'aut pas oublier, la meilleure école pour nos marins : c'est la que se formenl les malclots indispensables in notro flotte de guerre. Nénumoins, nous pollvons, dans certaines eonditions, souscrire a une transaction. Mais rien ne nous y foree. Il semble, qu'en lomme politique, l'Angleterre devrait s'inspirer de la constatution de cet itat de choses pour renoncer a des procidis diplomatiques qui ne sont pas dignes du rang ju'elle occupe parmi les nations. In France a fnit preuve, au cours le ce long dillivend, de la loyauté nhsolne qui est sa règle de conduite trailitionnelle; elle $n$ voulı croire a la bonne foi parfaite du fonvernement britannique. Il importe à celui-ci de ne pas la laisser s'ell repentir. Il y trouvern double avantnge : honneur et profit inatioricl.

\section{J. Cincenon,}

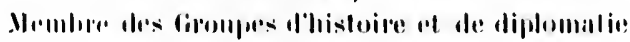

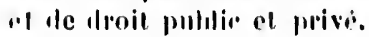





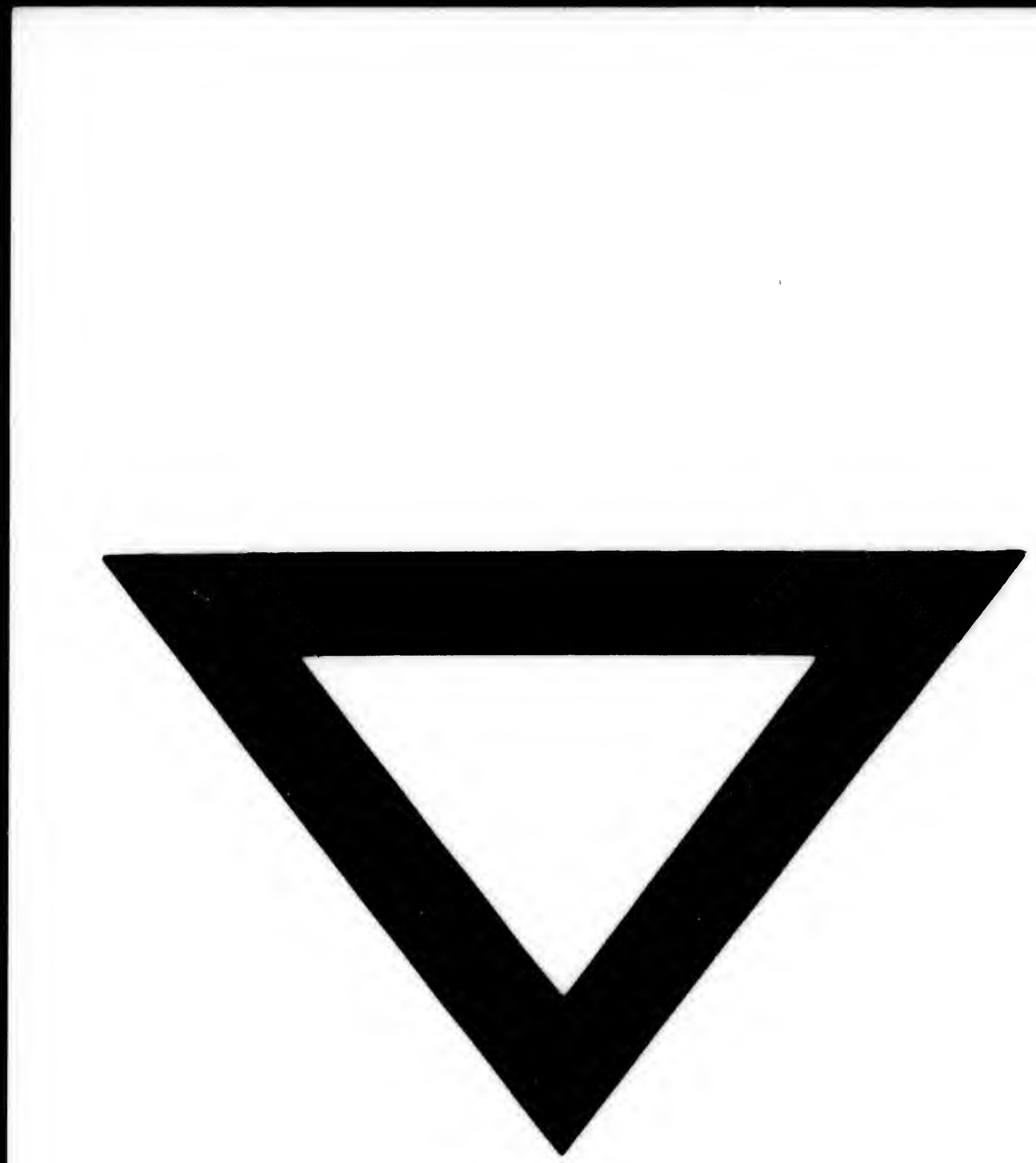

